BONPLANDIA 16(3-4): 279-284. 2007

\title{
NUEVAS CITAS EN RUBIACEAE DE BRASIL
}

\author{
ELSA L. CABRAL ${ }^{1}$, GIOVANNA FANECO PEREIRA² \& MARÍA CONCEICAO DE SOUZA ${ }^{3}$
}

\begin{abstract}
Summary: Cabral, E. L., Faneco Pereira, G. \& de Souza, M. C. 2007. New records in Rubiaceae of Brazil. Bonplandia 16(3-4): 279-284. ISSN: 0524-0476.

Galianthe guaranitica E.L. Cabral and G. parvula E.L. Cabral are reported for the first time in Brazil, Rosenbergiodendron Iongiflorum (Ruiz \& Pav.) Fagerl. and Guettarda pohliana Müll. Arg. are mentioned for the first time for Paraná State. Schwendenera tetrapyxis K. Schum., Galianthe dichasia (Sucre \& C. G. Costa) E.L. Cabral and Galianthe longisepala E.L. Cabral are new records for Parana, Mato Grosso do Sul and Mato Grosso States respectively.
\end{abstract}

Key words: Galianthe, Guettarda, Rosenbergiodendron, Schwendenera, Mato Grosso, Mato Grosso do Sul, Paraná, Brazil.

Resumen: Cabral, E. L., Faneco Pereira, G. \& de Souza, M. C. 2007. Nuevas citas en Rubiaceae de Brasil. Bonplandia 16(3-4): 279-284. ISSN: 0524-0476.

Galianthe guaranitica E.L. Cabral y G. parvula E.L. Cabral son primeros registros para Brasil, Rosenbergiodendron longiflorum (Ruiz \& Pav.) Fagerl. y Guettarda pohliana Müll. Arg. son mencionadas por primera vez para el estado de Paraná. Schwendenera tetrapyxis K. Schum., Galianthe dichasia (Sucre \& C. G. Costa) E.L. Cabral y Galianthe longisepala E.L. Cabral son nuevas citas para los estados de Paraná, Mato Grosso do Sul y Mato Grosso respectivamente.

Palabras clave: Galianthe, Guettarda, Rosenbergiodendron, Schwendenera, Mato Grosso, Mato Grosso do Sul, Paraná, Brasil.

Durante el relevamiento de las Rubiáceas de la "vegetación riparia" de un trecho del alto río Paraná y en el estudio de géneros de la tribu Spermacoceae de Brasil, se han hallado novedades para la flora brasileña, siete nuevas citas para los estados de Paraná, Mato Grosso y Mato Grosso do Sul, de las cuales dos son mencionadas por primera vez para Brasil.

\section{Galianthe Griseb.}

Es un género americano que se diferencia de los demás géneros de la tribu Spermacoceae, por las inflorescencias tirsoideas con flores dimorfas, por los frutos dehiscentes que pueden presentar mericarpos dehiscentes o indehis-

\footnotetext{
${ }^{1}$ Facultad de Ciencias Exactas, Físicas y Naturales y Agrimensura (UNNE), Instituto de Botánica del Nordeste, Casilla de Correo 209, 3400 Corrientes, Argentina. E-mail: ecabral@agr.unne.edu.ar

2 Programa de Pos-graduação em Ambientes Aquaticos Continentales (PEA), UEM, Maringá, Parana, Brasil.

${ }^{3}$ Universidad Estadual de Maringá (UEM)-Nupélia. Dep. Biologia, Maringá, Parana, Brasil.
} 
centes y por los granos de polen colporados, con exina de retículo complejo. Consta de 49 especies y en Brasil se encuentra el principal centro de concentración y diversidad del género, representado por 37 especies, de las cuales 20 son endémicas (Cabral, 2002).

\section{Galianthe dichasia (Sucre \& C.G. Costa) E.L. Cabral}

Fig. $1 \mathrm{G}$ y H

Cabral, E. L., Bol. Soc. Argent. Bot. 27: 242. 1991.

Borreria dichasia Sucre \& C. G. Costa, Loefgrenia 48: 2. 1970.

Diodia cymosa Cham. var. aculeolata Hassl., Feddes Repert. Spec. Nov. 14: 168. 1915.

Sufrútice de 50-80 cm alt., tallos basales radicantes, fistulosos, alados. Inflorescencia cimoidal, dicasial a monocasial, con inflorescencias parciales subglomeriformes, flores dimorfas.

Referencia: Cabral \& Bacigalupo (1997), Cabral (2002).

Distribución y ecología. Su área abarca Paraguay (Alto Paraná, Guairá, San Pedro), Uruguay (Rivera), Argentina (Misiones), SE del Brasil (Paraná, Sta. Catarina y Rio Grande do Sul) y recientemente fue coleccionada en el Estado de Mato Grosso do Sul. Vive en terrenos bajos, pantanosos y orilla de vertientes.

Fenología: Florece y fructifica de diciembre a marzo.

Material estudiado: BRASIL. Mato Grosso do Sul. Mun. Taquarussu, Ribeirão Vitório, 15-II2006, G. F. Pereira \& M. C. Souza 153 (HUEM); Jateí, rio Curupaí, 03-V-2006, G. F. Pereira 172 e 173 (HUEM).

\section{Galianthe guaranitica (Chodat \& Hassl.) E.L. Cabral}

Cabral, E. L, Bol. Soc. Argent. Bot. 27: 244. 1991.
Borreria guaranitica Chodat \& Hassl., Bull. Herb. Boissier, sér. 2, 4: 186.1904.

Sufrútice con xilopodio de 0,5-1,5 m alt., tallos simples o escasamente ramificados. Hojas elípticas, ápice de agudo a atenuado, base redondeada o levemente cordada Inflorescencias tirsoideas terminales, \pm comprimidas, con flores dimorfas.

Referencia: Cabral (1991), Cabral (2002).

Distribución y ecología: Esta especie estaba registrada para un área restringida de Paraguay, en los departamentos Amambay y Canindeyú (Cabral, 1991). Una colección posterior permitió ampliar el área al Estado de Mato Grosso do Sul en Brasil. Habita en campos cerrados con suelos arenosos, graminosos, sujetos a quemazones periódicas, 500-700 m.s.m.

Fenología: Florece y fructifica de diciembre a abril.

Material estudiado: BRASIL. Mato Grosso do Sul. Mun. Sidrolandia, Santa Fe, 23-I-1971, G. Hatschbach 26028 (CTES, MBM, NY, US).

\section{Galianthe longisepala E. L. Cabral}

Cabral, E. L., Bonplandia 13: 15. 2004.

Sufrútice con xilopodio de 15-25 (-30) cm alt., ramas secundarias poco desarrolladas o ausentes. Hojas lanceoladas o elípticolanceoladas, glabras en ambas caras, discoloras, con 3-4 pares de nervios secundarios, en material herborizado de color marrónnegruzcos en el envés. Inflorescencias congestas en todas las ramas. Cáliz con 4 segmentos reflexos en el fruto. Corola blanca. Cápsula subglobosa, glabra, con notables sépalos reflexos.

Distribución geográfica y ecología: Galianthe longisepala vive en campos cerrados, en suelos lateríticos y con afloramiento rocosos entre 1050-1250 m s. m. Cabral (2004) señala que habita en los Estados de Goiás y Minas Gerais; con el material recien- 


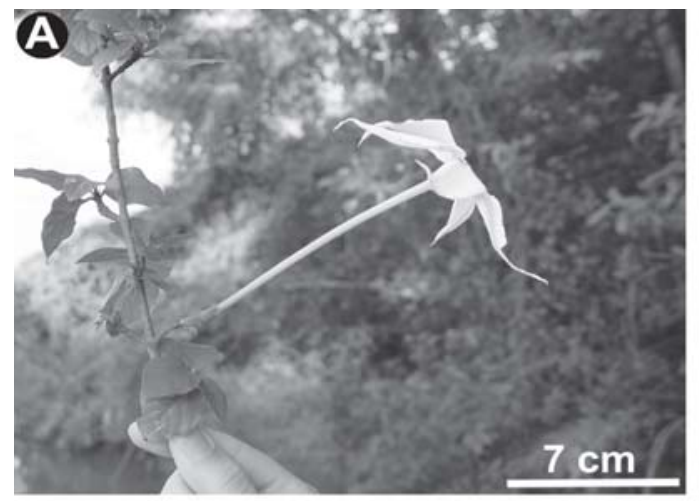

C
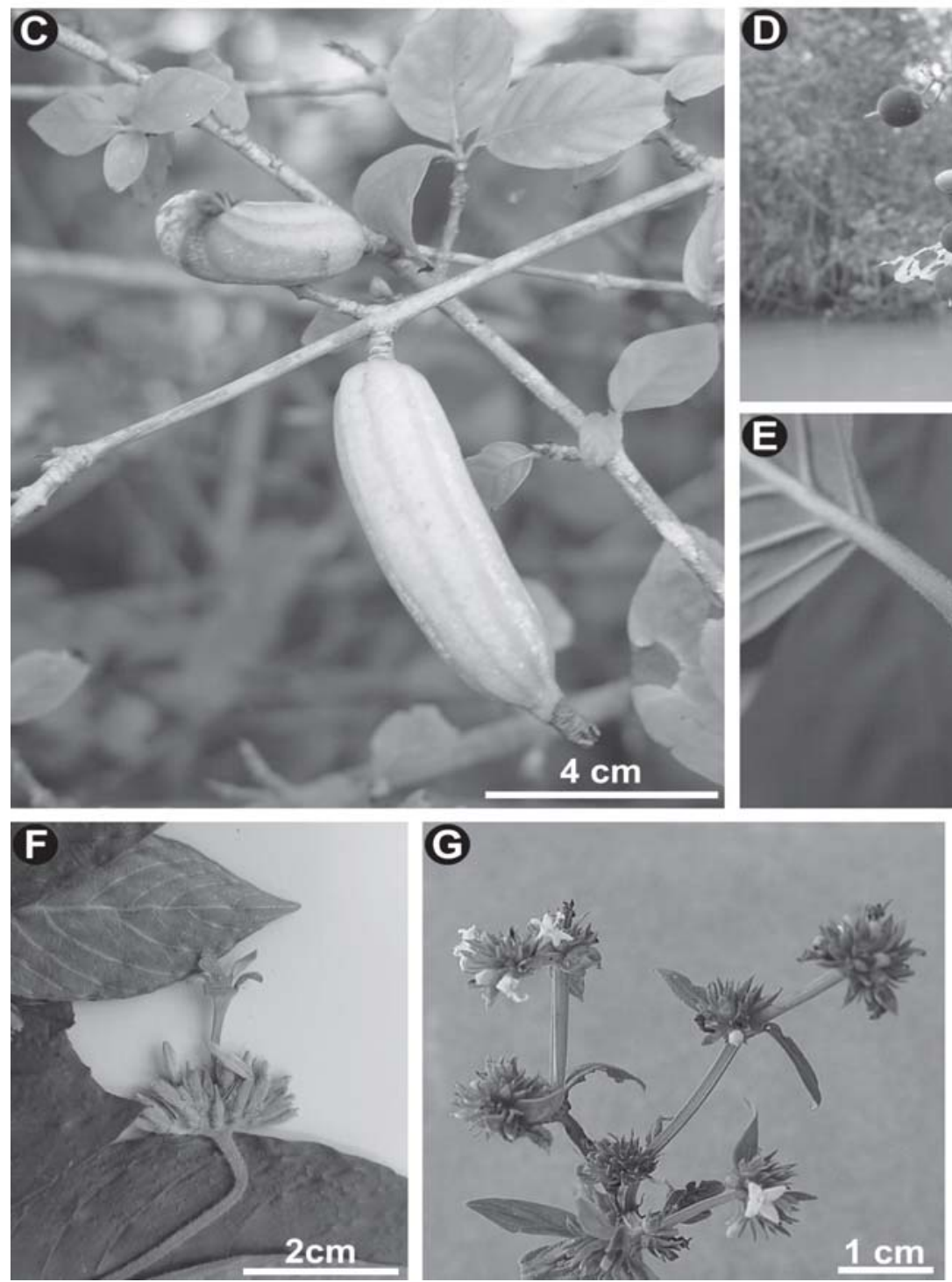

G
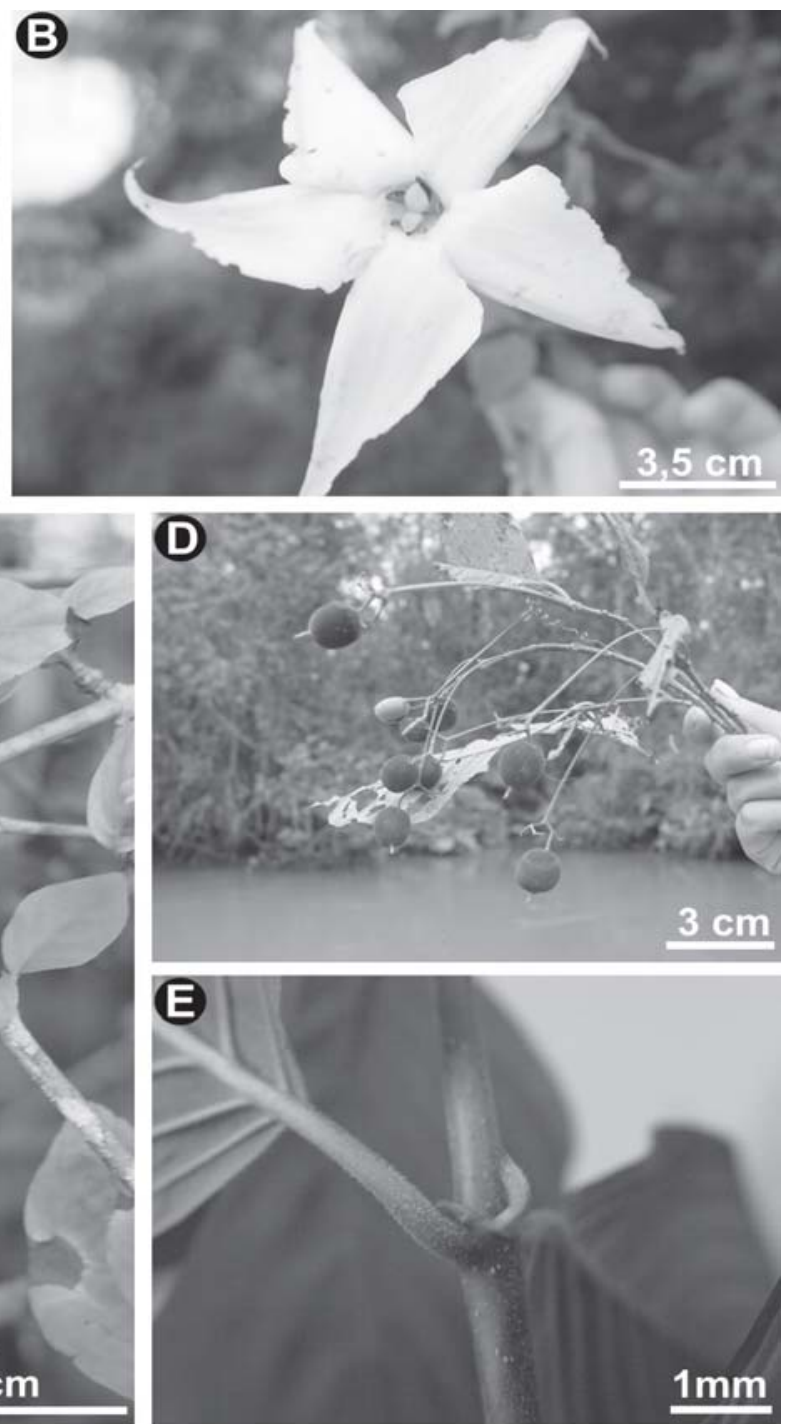

Fig. 1. Rosenbergiodendron longiflorum (Ruiz \& Pav.) Fagerl. A-B: flor. C: rama fructífera. Guettarda pohliana Müll. Arg. D: rama fructífera. E: estípula. F: inflorescencia. Galianthe dichasia (Sucre \& C.G. Costa) E.L. Cabral. GH: inflorescencia (fotos de G. Faneco Pereira) 
temente identificado se amplía su distribución al Estado de Mato Grosso.

Fenología: Florece y fructifica de enero a marzo.

Obs.: Esta especie se reconoce fácilmente, por las hojas con la nervadura obscura, contrastante con el color de la lámina en el envés, por las inflorescencias densas y por los frutos con sépalos largos y reflexos.

Material estudiado: BRASIL. Mato Grosso. Mun. Chapada dos Guimaraes, Veu do Noiva e Cachoeirinha, 19-III-1983, P. Lisboa \& al. 3077 (MG).

\section{Galianthe parvula E. L. Cabral}

Cabral, E. L., Bonplandia 7: 24. 1993.

Sufrútice con xilopodio, muy ramificado de 20-25 (-40) cm alt. Inflorescencias cortas, congestas, en las ramas principales y secundarias. Cápsula globosa y pilosa. Semilla con el estrofíolo caduco, que persiste en el tabique interlocular del fruto.

Referencia: Cabral (1993), Cabral (2002).

Distribución y ecología: Se la conocía para Paraguay (Dep. Amambay, San Pedro) y recientemente se estudió material de Brasil, del Estado Mato Grosso do Sul. Vive en campos cerrados, campos rocosos y en laderas de colinas.

Fenología: Florece de octubre a enero, fructifica de febrero a abril.

Material estudiado: BRASIL. Mato Grosso do Sul. Mun. Ponta Pora, $16 \mathrm{~km}$ do estrada a Campo Grande, 22³2'0”S 5541'0” W, cerrado, 18 Nov. 1963, J. Correa Gomes Jr. 1437 (UB).

\section{Guettarda L.}

Es un género de la tribu Antirheoideae, con cerca de 80 especies de amplia distribución en América tropical (Taylor \& Steyermark, 2004).

\section{Guettarda pohliana Müll. Arg.}

Fig. $1 \mathrm{D}-\mathrm{F}$

Müller Argoviensis, J., Flora 58: 450. 1875.

Matthiola pohliana (Müll. Arg.) Kuntze, Rev. Gen. Pl. 1: 288. 1891.

Arbusto espinoso de aproximadamente $3 \mathrm{~m}$ alt., con tallos cerosos, lenticelados, los más jóvenes pilosos. Hojas discoloras, amarillentas, nervios marcados. Inflorescencias cimosas, multifloras, axilares. Frutos carnosos, oblongos, colorados, velutinos, con cáliz persistente.

Referencia: Müller Argoviensis (1881).

Distribución y ecología: Se conocía para los estados de Distrito Federal, Goiás, Minas Gerais, São Paulo y recientemente se la coleccionó en el estado de Paraná. Crece en la selva riparia.

Fenología: Florece y fructifica de abril a octubre.

Material estudiado: BRASIL. Paraná: Porto Rico, Rio Paraná, Ilha Mutum, 08-XII-1993, fl., M. C. Souza \& M. Sobral 15 (HUEM); remanescente entre a base e a cidade, 16-I-1994, fl., fr., M.C. Souza 616 (HUEM).

\section{Rosenbergiodendron Fagerl.}

Género neotropical perteneciente a la tribu Gardenieae, afín a Randia L., donde estaba incluido, del que se diferencia por presentar polen en mónades en flores perfectas, con coléteres internos y comprender plantas inermes, versus polen en tétrades en flores imperfectas, sin coléteres internos, y constituir plantas espinosas (Gustaffsson, 1998). Consta de tres especies, $R$. densiflorum (K. Schum.) Fagerl., R. fomosum (Jacq.) Fagerl. y $R$. longiflorum (Ruiz \& Pav.) Fagerl.; la última 
es la única que vive en el área estudiada.

\section{Rosenbergiodendron longiflorum (Ruiz \& Pav.) Fagerl.}

Fig. 1 A, B y C

Fagerlind, F., Svensk. Bot. Tidskr. 42: 152. 1948. Gardenia longiflora Ruiz \& Pav., Fl. Peruv. 2: 67, Ic. 219.1799.

Randia ruiziana DC., Prodr. 4: 388. 1830.

Randia speciosa DC., Prodr. 4: 388. 1830.

Randia formosa var. longiflora (Ruiz \& Pav.) K. Schum., Fl. Bras. 6(6): 343. 1889.

Basanacantha macrocarpa Rusby, Mem. New York Bot. Gard. 7: 374. 1927.

Randia williamsii Standl., Publ. Field Columbian Mus., Bot. Ser. 8: 169. 1930.

Subarbusto o arbolito de 1-7 m alt., que se diferencia fácilmente de las otras dos especies del género, por poseer el tubo de la corola notablemente largo $(12-30 \mathrm{~cm})$ y el fruto elipsoidal a cilíndrico, de 5-10 cm long.

Referencia: Gustaffsson (1998), Taylor \& Steyermark (2004).

Distribución y ecología: Habita en Bolivia, Colombia, Ecuador, Guayana, Perú y en Brasil se halla ampliamente distribuida en el nordeste, en los Estados de Acre, Amapá, Amazonas, Goiás, Maranhão, Mato Grosso, Pará, y es citada por primera vez para el Estado de Paraná. Crece en la selva a orillas de los ríos.

Fenología: Florece y fructifica de abril a octubre.

Material estudiado: BRASIL. Paraná: Porto Rico, Rio Paraná, Canal Cortado, 07-VI-1992, fl., fr., R. Pilati 55 (HUEM); 17-V-1994, fl., fr., M. Curti 69 (HUEM); Ilha Japonesa, 31-V-2001, fl., fr., M.B. Romagnolo s.n. (HUEM); Mata do Araldo, 23-IX1992, M. C. Souza 356 (HUEM).

\section{Schwendenera K. Schum.}

Es un género monotípico de la tribu
Spermacoceae, que se diferencia de los géneros más afines, por presentar flores dimorfas y frutos esquizocárpicos, con cuatro mericarpos indehiscentes, con segmentos del cáliz persistentes en cada uno de ellos.

\section{Schwendenera tetrapyxis K. Schum.}

Schumann, K., Sitzungsber Ges. Naturf. Freunde, Berlin1886: 157. 1887.

A esta especie se la conocía sólo por el ejemplar tipo (São Paulo, Riedel 1879) y después de más de cien años, se realizó otra colección en el mismo estado. Recientemente se halló material proveniente del estado de Paraná, por lo cual se extiende su distribución geográfica.

Referencia: Schumann (1888); Bacigalupo \& Cabral (2007).

Fenología. Florece y fructifica de septiembre a marzo.

Material estudiado: BRASIL. Paraná. Mun. Dr. Camargo, Rio Ivai, 16-IX-1969, G. Hatschbach 21526 (MBM).

\section{Bibliografía}

BACIGALUPO, N. M. \& E. L. CABRAL. 2007. Tribu Spermacoceae. In M. Wanderley, G. Shepherd, T. Melhem, \& A. M. Giulietti (eds.). Flora Fanerogamica do estado de São Paulo 5.

CABRAL, E. L. 1991. Rehabilitación del género Galianthe (Rubiaceae). Bol. Soc. Argent. Bot. 27: 235-249.

- 1993. Novedades en Galianthe (Rubiaceae). Bonplandia 7: 24-25.

— 2002. Revisión del género Galianthe (Rubiaceae). Tesis doctoral, Universidad Nacional del Nordeste, Corrientes.

2002. Dos especies nuevas de Galianthe (Rubiaceae) para Brasil. Bonplandia 13: 15-19.

— \& N. M. BACIGALUPO. 1997. Revisión del género Galianthe subg. Ebelia, stat. nov. (RubiaceaeSpermacoceae). Ann. Missouri Bot. Gard. 83: 857- 
877.

GUSTAFSSON, C. G. R. 1998. The neotropical Rosenbergiodendron (Rubiaceae, Gardenieae). Brittonia 50(4): 452-466.

MÜLLER ARGOVIENSIS, J. 1881. Rubiaceae, tribu Guettardeae. In C. F. P Martius (ed.). Flora brasiliensis 6 (5): 23-24.
SCHUMANN, K. 1888. Rubiaceae. In C. F. P. Martius (ed.). Flora brasiliensis 6(6A): 98-99.

TAYLOR, C.M. \& J. A. STEYERMARK. 2004. Guettarda. In J. A. Steyermark, P. E. Berry, K. Yatskievych \& B. Holst (eds.). Flora of the Venezuelan Guayana 8: 614. Missouri Botanical Garden Press, Saint Louis.

Original recibido el 12 de julio de 2007; aceptado el 10 de noviembre de 2007. 